\title{
Self-efficacy, Physical Activity and QOL in People with MS
}

Cecilie Fjeldstad* and Gabriel Pardo

OMRF Multiple Sclerosis Center of Excellence, 820 NE 15th Street, Oklahoma City, USA

\begin{abstract}
Self-Efficacy is part of the social-cognitive theory defined as the belief that one can successfully cope with challenging situations and attain certain goals. It has been suggested this principle can be applied to physical and psychological quality of life in individuals with Multiple Sclerosis (MS).

Objective: To examine if self-efficacy and physical activity have relationships with quality of life (QOL) in individuals with MS.

Methods: 109 individuals with MS participated in this study. Each individual completed the Multiple Sclerosis Self-Efficacy scale (MSSE), the Multiple Sclerosis Impact Scale (MSIS-29), and the Good in Leisure-Time Exercise Questionnaire (GLTEQ). Pearson product moment correlation coefficients were computed for self-efficacy, physical activity and QOL.

Results: The sample $(n=109)$ was composed as follows, females $(75 \%)$, relapsing remitting form of MS $(81 \%)$, married $(68 \%)$, employed $(44 \%)$. Time since MS diagnosis was 7.6 years $(\mathrm{SE}=0.62)$. There were moderately high negative correlations between MSSE and QOL physical component $(r=-0.65, p<0.01)$ and psychological component $(r=-0.63, p<0.01)$, indicating that individuals with increased sense of self-efficacy experienced less psychological issues and an increased level of participation in physical tasks. There was a low negative but significant correlation between total time spent in leisure activity and $\mathrm{QOL}$ physical component $(r=-0.21, p<0.05)$, but not for $\mathrm{QOL}$ psychological component. Physical activity has a negative correlation with physical impact of QOL $(r=-0.21, p<0.05)$ and no correlation with psychological component ( $p>0.05)$.
\end{abstract}

Conclusion: The results of this study demonstrate that with increased self-efficacy there is an increase in QOL on both physical and psychological components, which is important for increased independence and functionality in individuals with MS.

Keywords: Multiple sclerosis; Self-efficacy; Quality of life; Physical activity

\section{Introduction}

Multiple Sclerosis (MS) is an immune-mediated disease of the central nervous system (CNS) with both inflammatory and degenerative components. It can present with an array of symptoms such as visual disturbances, motor and sensory deficits, bladder and sexual dysfunction, and psychological issues to include depression, anxiety, and cognitive dysfunction [1].

The diagnosis of MS, given the chronic nature of the disease and the fact that it implies an un-predictable path to variable degrees of disability, can have a great psychological impact and a distressing effect on quality of life (QOL). Given the variety and severity of symptoms in MS, often not visible to other people, coping mechanisms may be compromised and disrupt many aspects of life from personal (family and relationships) to professional (employment). This may impact an individuals' self-efficacy.

\section{Self-efficacy in MS}

Self-Efficacy is part of the social-cognitive theory that involves beliefs that one can successfully cope with challenging situations and have the ability to overcome them [2]. An individual's level of selfefficacy is often influenced by their accomplishments or capacity of performance, verbal persuasion, and the interpretation of psychological and affective states [2]. Individuals with strong self-efficacy beliefs will more likely set higher personal goals believing they will successfully achieve them. In contrast, someone with low self-efficacy beliefs will be more likely to set lower personal goals, as they do not believe they will be able to achieve high objectives. In the context of a chronic illness, their beliefs may affect their own perception of the condition, the impact the illness will have on their life, and to which capacity they are able to handle the disease. Previous studies have shown high self-efficacy to be a predictor of health related behavior such as adherence to medication $[3,4]$, decreased depression $[5,6]$ and increased participation in physical activity [7-11]. Self-efficacy has been demonstrated to be a predictor of psychological improvement in individuals with chronic diseases such as cancer [12], rheumatoid arthritis [13,14], fibromyalgia [15] and MS $[16,17]$.

\section{Physical activity in MS}

Participation in physical activity (PA) of individuals diagnosed with MS is generally low [18], especially in those that complain of multiple or worsening of symptoms $[9,19]$ and those that report high levels of fatigue or difficulty walking [20]. In the past, individuals diagnosed with MS were recommended not to engage in any physical activity in order to avoid even a slight increase in core body temperature which may worsen neurological function, known as Uhthoff's phenomenon [21]. More recently it has been demonstrated that individuals with MS benefit from physical activity in a similar way to the non-diseased

*Corresponding author: OMRF Multiple Sclerosis Center of Excellence, 820 NE 15th Street, Oklahoma City, USA, Tel: 405.271.8001, extn 58089; Fax: 405.271.2319; E-mail: Cecilie-Fjeldstad@omrf.org

Received January 21, 2014; Accepted February 24, 2014; Published March 01, 2014

Citation: Fjeldstad C, Pardo G (2014) Self-efficacy, Physical Activity and QOL in People with MS. J Neurol Neurophysiol 5: 194. doi:10.4172/2155-9562.1000194

Copyright: @ 2014 Fjeldstad C, et al. This is an open-access article distributed under the terms of the Creative Commons Attribution License, which permits unrestricted use, distribution, and reproduction in any medium, provided the original author and source are credited. 
population [22] and experience improvement in their fatigue levels [23]. Participation in physical activity can be greatly compromised in the MS population as the disease presents with both physical (spasticity, muscle weakness, ataxia, fatigue, and pain), and psychological (emotional state, anxiety and depression) symptoms that decrease the ability and willingness to do so.

\section{Quality of life in MS}

Quality of life (QOL) is affected in a large number of individuals living with chronic diseases like MS [24,25]. The unpredictable clinical course and absence of a curative treatment in MS greatly affect the psychological QOL, with about 50\% of individuals experiencing depression at some point during the course of the disease [26]. Individuals with self-reported more rapidly progressive types of MS usually experience greater disability and impairment, and thus often have lower QOL [27]. However, one study showed that duration of disease positively correlated with better QOL, despite greater physical disability as measured by expanded disability status scale (EDSS) [2830], possibly due to the capacity to adjust to the effects of the disease and accept limitations over time. Participation in physical activity has shown to improve QOL in MS individuals $[29,30]$ with the most active individuals reporting higher levels of health related QOL (HRQOL) on both physical and mental components [31].

\section{Purpose}

The purpose of this study is to identify the relationship between physical activity level and self-efficacy with components of QOL. Our hypothesis is that individuals with low self-efficacy would also report decreased quality of life and have less participation in physical activities. Understanding this process can lead to interventions that will offer better results in the comprehensive management of MS by identifying barriers to patient's participation in their own care.

\section{Material and Methods}

\section{Subjects}

Patients were recruited from a single specialized MS center at the time of scheduled office visits and data was collected as part of routine clinical assessments. The sample was a convenience sample and consisted of a total of 109 subjects with diagnosis of MS following 2010 McDonald criteria [32], with mean age 45 years, height $168 \mathrm{~cm}$, weight $80 \mathrm{~kg}$ and mean duration since diagnosis of 7.6 years. The sample was $75 \%$ females, $68 \%$ married, $44 \%$ employed, $40 \%$ with partial college education, and $38 \%$ being college graduates. Of the 109 individuals, $81 \%$ had relapsing remitting MS (RR-MS); 14\% had secondary progressive MS (SP-MS); 5\% had primary progressive (PP-MS).All of them were ambulatory without aids but ankle-foot orthosis and peroneal nerve electric stimulators to compensate for foot drop were allowed.

The study protocol was approved by the institutional review board, and all participants in the study provided written informed consent prior to data collection. All subjects had the cognitive ability to read and sign informed consent as determined by clinical assessment of the treating neurologist.

\section{Protocol for acquisition}

Each individual completed the following questionnaires: Multiple Sclerosis Self-Efficacy scale (MSSE) [33], a disease-specific 14-item questionnaire with a 6-point scale, ranging from 1 (strongly disagree) to 6 (strongly agree). The MSSE has been proven to be reliable and is a useful tool in assessment of psychological adjustments in both clinical and research settings [33]. Total scores range from 14 to 84 , where a higher score indicates an elevated level of self-efficacy.

The Multiple Sclerosis Impact Scale (MSIS-29), a disease-specific, self-administered questionnaire asking about the day to day impact that MS has had on life for the immediate previous two weeks. It includes physical (20 items) and psychological (9 items) subscales [34], and is proven to be a useful tool in the assessment of disease impact and quality of life in community [34], hospital setting [35] and in clinical research [36]. It is a 29 -item questionnaire and is scored as follows: $1=$ not at all, $2=$ a little, $3=$ moderately, $4=$ quite a bit, $5=$ extremely, for evaluating the degree of an experienced problem the last two weeks. The score ranges from minimum of 29 to a maximum of 145 , with higher scores indicating greater impact of the disease on the individual's QOL.

The Good in Leisure-Time Exercise Questionnaire (GLTEQ) [37], is a simple generic measure of physical activity that is widely used in clinical and epidemiological settings. It is a self-administered twoitem questionnaire that measures an individual's physical activity level, with the first question determining the average number of times an individual participated in strenuous (e.g., jogging), moderate (e.g., fast pace walking) and mild exercise (e.g., easy pace walking), for more than 15 minutes during their free time in a typical 7-day period. These frequencies are then multiplied by nine, five, and three metabolic equivalents respectively and then summed to a total single measure of physical activity. In this particular study, we only included the first question in the analysis, as it provides more information of the amount of PA, as done in previous research [38]. The second question is ordinal in nature and has three options ( $1=$ often, $2=$ sometimes, $3=$ never/rarely) and measures how often the individual engages in any regular activity long enough to work up a sweat or rapid beating of the heart. Evidence has shown that GLTEQ gives a valid measure of physical activity in individuals diagnosed with MS [39].

\section{Data analysis}

Descriptive analyses are presented as mean \pm standard errors and were carried out for all variables in the group. The relationship among scores from the three measures was examined using Pearson product moment correlation coefficients. All statistical analysis was performed using SPSS, version 16.0 (SPSS Inc, Chicago, IL, USA). Statistical significance was set at $\mathrm{p} \leq 0.05$. Demographic characteristics of the sample are displayed in Table 1.

\section{Results}

\section{Self-efficacy (MSSE) and quality of life (MSIS-29) psychological and physical impact}

A moderate negative correlation between MS self-efficacy (MSSE) and quality of life (MSIS-29) psychological impact of MS ( $r=-0.63$, $\mathrm{p}<0.01)$ was demonstrated, indicating that as levels of self-efficacy increase, the psychological impact on QOL decreases (Figure 1). There was a moderate negative correlation between self-efficacy and physical impact of MS ( $r=-0.65, \mathrm{p}<0.01)$, with greater self-efficacy being associated with a lesser impact of physical deficits on QOL (Figure 2).

\begin{tabular}{|c|c|}
\hline Age $(\mathrm{yrs})$ & $45.05 \pm 1.13$ \\
\hline Height $(\mathrm{cm})$ & $168.00 \pm 0.01$ \\
\hline Weight $(\mathrm{kg})$ & $79.70 \pm 2.33$ \\
\hline MS yrs & $7.62 \pm 0.62$ \\
\hline
\end{tabular}

Mean \pm SE, MS; multiple sclerosis;

Table 1: Subject Characteristics $(n=109)$. 


\section{Physical activity and MSIS-29 psychological and physical impact}

A weak negative correlation was found between total time spent in leisure time physical activity and quality of life physical impact $(\mathrm{r}=-0.21, \mathrm{p}<0.05)$, suggesting the less physical impact MS had on individuals, the more time they spent in physical activity, (Figure 3).No correlation of significance was found for time spent in physical activity and psychological impact of MS ( $\mathrm{p}>0.05$ ) (Figure 4).

\section{Discussion and Conclusion}

This study provided information of MS self-efficacy and the time spent in physical activity and its effect on quality of life, both physical and mental, in MS individuals. A moderate relationship was evident, with those individuals having increased levels of self-efficacy reporting a lesser mental and physical impact of their underlying disease on QOL.A weaker relationship was found between time spent participating in physical activity and the physical impact component of quality of life.

Participation in physical activity may help minimize some of the symptoms associated with MS, to include fatigue, ambulation and postural balance, due to increased muscle strength [40]. It may also help decrease the risk of developing secondary diseases that result from sedentary lifestyles and inactivity such as cardiovascular disease

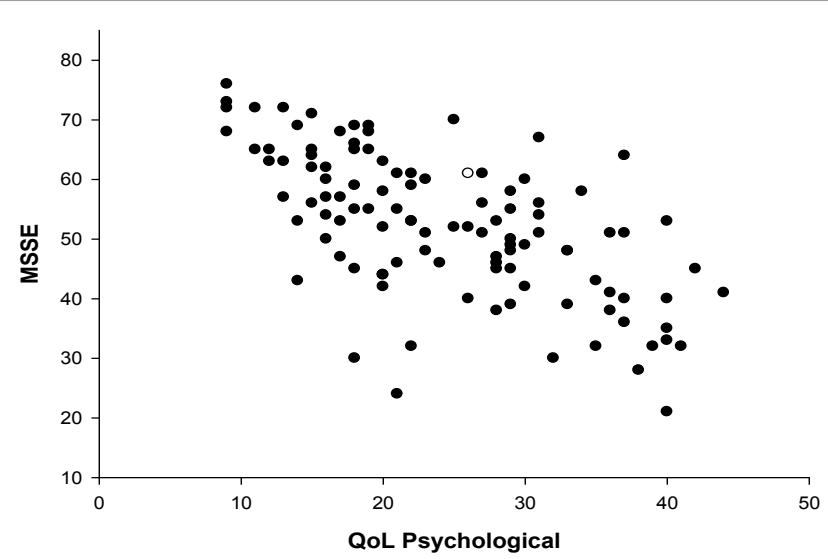

Figure 1: Self-efficacy (MSSE) and QOL (MSIS-29) psychological impact of MS.

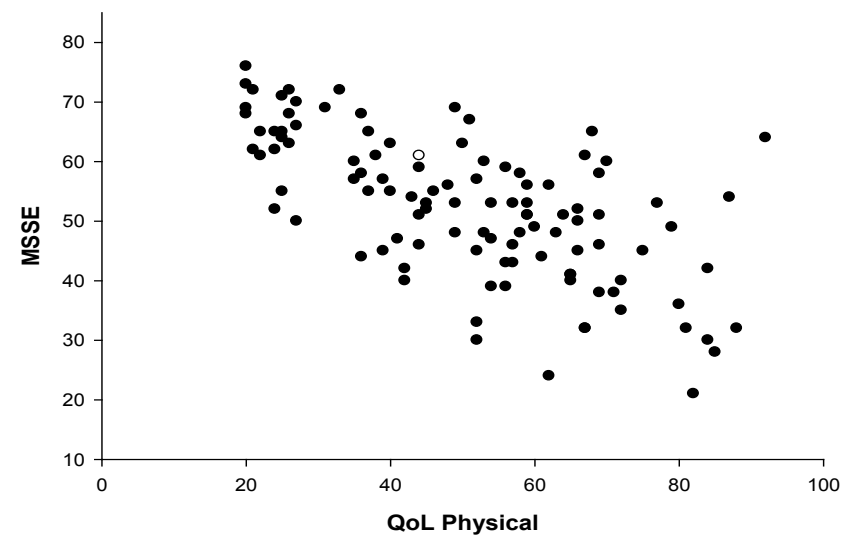

Figure 2: Self-efficacy (MSSE) and QOL (MSIS-29) physical impact of MS.

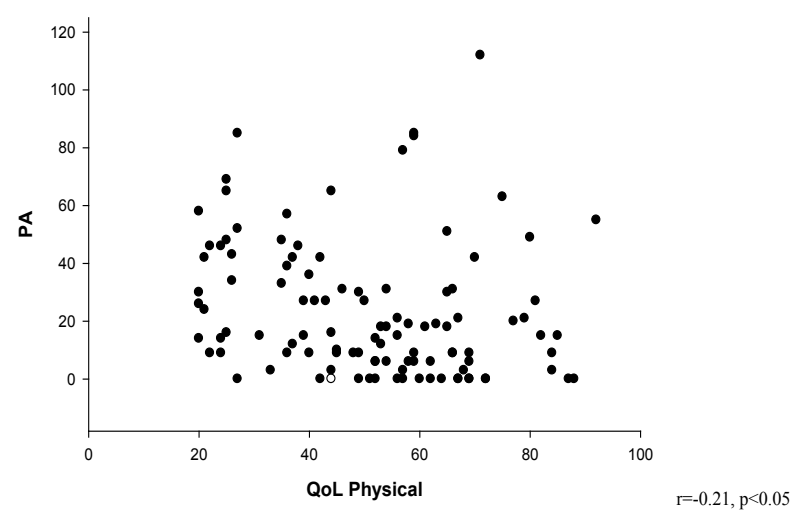

Figure 3: Time spent in physical activity (PA) and QOL (MSIS-29) physical impact of MS.

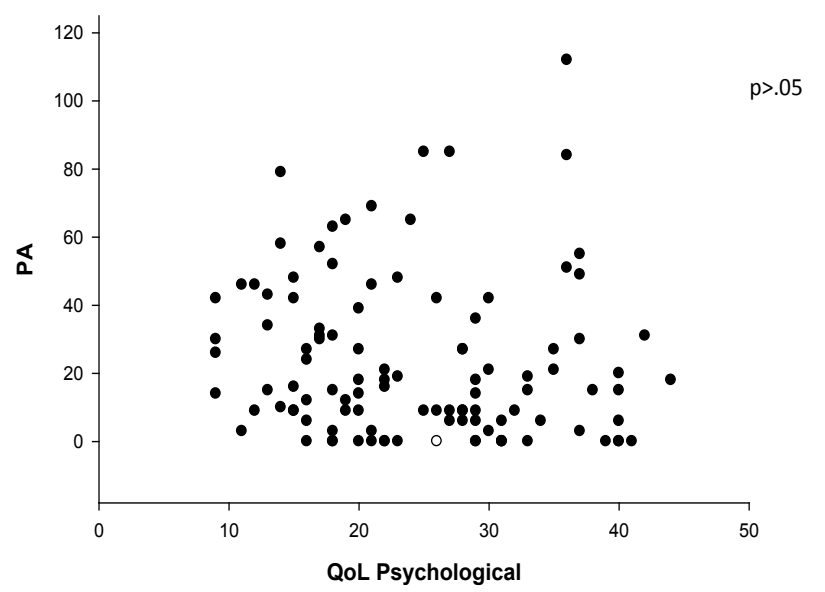

Figure 4: Time spent in physical activity (PA) and QOL (MSIS-29) psychological impact of MS.

and osteoporosis. Many factors influence the ability and willingness to engage in regular physical activity in MS. An individualized program that adapts to the participant's limitations is recommended. Some modalities include aquatics and resistance training for MS individuals affected by thermo-sensitivity, as the core temperature will not increase as much with this type of exercise as it does with others such as running.

Quality of life is commonly compromised in MS individuals. Studies have established that QOL can be influenced by factors such as self-efficacy and physical activity, with an increase in quality of life often seen in individuals that report greater self-efficacy and more participation in physical activity [41]. A recent study showed that selfefficacy is a significant predictor of self-reported physical, cognitive and social function [42]. Those results coincide with some of the results of this study, where we demonstrated that individuals with greater selfefficacy reported less psychological and physical impact from MS in their QOL. In addition, this investigation found that individuals that spent more time participating in physical activity reported less physical impact of MS and thus increased physical independence. This positive effect was not found for time spent in physical activity and psychological impact. 
There are many ways to obtain measures of physical activity levels in individuals with MS. In this study, amount of time spent exercising was measured by self-report questionnaires and not by objective measures often used by other researchers $[43,44]$. One previous study was able to detect differences in physical activity levels between MS individuals compared to a sedentary control group by the use of accelerometers but did not find any significant difference by self-report in the same study [44]. The use of both objective means and self-report as measures of physical activity has been previously validated [39].

The predominance of females (75\%) in this study reflects the demographics of MS. Two of the 3 instruments used in this study are disease-specific, making the results not generalizable to other chronic entities.

In conclusion, this study showed that with greater self-efficacy there is a decrease in the amount of impact MS has on the individual's physical and psychological components of QOL. This is important for overall increased independence and functionality in the MS population. Determining self-efficacy reveals important information regarding how individuals handle, cope and adjust to having a chronic disease like MS, influencing participation in self-care and disease management. It is important to educate MS patients about the benefit of physical activity to promote better, healthier and functional independent lifestyles. Selfefficacy should be incorporated in the comprehensive assessment and management of MS to improve long-term outcomes.

\section{References}

1. Society NMS (2005) Multiple sclerosis information sourcebook, I.R.C.a.L.o.t.N.M.S. Society, Editor New York, NY, USA.

2. Bandura A (1997) Self-Efficacy: The exercise of Control New York, Freeman, NY, USA.

3. Kavanagh DJ, Gooley S, Wilson PH(1992) Prediction of adherence and control in diabetes. J Behav Med 16: 1-14.

4. Zwibel H, Pardo G, Smith S, Denney D, Oleen-Burkey M (2011) A multicenter study of the predictors of adherence to self-injected glatiramer acetate for treatment of relapsing-remitting multiple sclerosis. J Neurol 258: 402-411.

5. Ehrenberg MF, Cox DN, Koopman RF (1991) The relationship between selfefficacy and depression in adolescents. Adolescence 26: 361-374.

6. Holahan CK, Holahan CJ (1987) Self-efficacy, social support, and depression in aging: a longitudinal analysis. J Gerontol 42: 65-68.

7. McAuley E (1993) Self-efficacy and the maintenance of exercise participation in older adults. J Behav Med 16: 103-113.

8. Trost SG, Owen N, Bauman AE, Sallis JF, Brown W (2002) Correlates of adults' participation in physical activity: review and update. Med Sci Sports Exerc 34: 1996-2001.

9. Motl RW, Snook EM, McAuley E, Gliottoni RC (2006) Symptoms, self-efficacy, and physical activity among individuals with multiple sclerosis. Res Nurs Health 29: 597-606.

10. Booth ML, Owen N, Bauman A, Clavisi O, Leslie E (2000) Social-cognitive and perceived environment influences associated with physical activity in older Australians. Prev Med 31: 15-22.

11. Sternfeld B, Ainsworth BE, Quesenberry CP (1999) Physical activity patterns in a diverse population of women. Prev Med 28: 313-323.

12. Cunningham AJ, Lockwood GA, Cunningham JA (1991) A relationship between perceived self-efficacy and quality of life in cancer patients. Patient Educ Couns 17: 71-78.

13. Barlow JH, Williams B, Wright C (1996) The Generalized Self-Efficacy Scale in people with arthritis. Arthritis Care Res 9: 189-196.

14. Schiaffino KM, Revenson TA, Gibofsky A (1991) Assessing the impact of selfefficacy beliefs on adaptation to rheumatoid arthritis. Arthritis care and research : the official journal of the Arthritis Health Professions Association 4: 150-157.
15. Buckelew SP, Huyser B, Hewett JE, Parker JC, Johnson JC, et al. (1996) Selfefficacy predicting outcome among fibromyalgia subjects. Arthritis Care Res 9: 97-104.

16. Barnwell AM, Kavanagh DJ (1997) Prediction of psychological adjustment to multiple sclerosis. Soc Sci Med 45: 411-418.

17. Wassem R (1992) Self-efficacy as a predictor of adjustment to multiple sclerosis. J Neurosci Nurs 24: 224-229.

18. Motl RW, McAuley E, Snook EM (2005) Physical activity and multiple sclerosis: a meta-analysis. Mult Scler 11: 459-463.

19. Motl RW, Arnett PA, Smith MM, Barwick FH, Ahlstrom B, et al. (2008) Worsening of symptoms is associated with lower physical activity levels in individuals with multiple sclerosis. Mult Scler 14: 140-142.

20. Motl RW, Snook EM, Schapiro RT (2008) Symptoms and physical activity behavior in individuals with multiple sclerosis. Res Nurs Health 31: 466-475.

21. Selhorst JB, Saul RF (1995) Uhthoff and his symptom. J Neuroophthalmol 15: 63-69.

22. Brown TR, Kraft GH (2005) Exercise and rehabilitation for individuals with multiple sclerosis. Phys Med Rehabil Clin N Am 16: 513-555.

23. White LJ, McCoy SC, Castellano V, Gutierrez G, Stevens JE, et al. (2004) Resistance training improves strength and functional capacity in persons with multiple sclerosis. Mult Scler 10: 668-674.

24. Benito-León J, Morales JM, Rivera-Navarro J, Mitchell A (2003) A review about the impact of multiple sclerosis on health-related quality of life. Disabil Rehabil 25: $1291-1303$.

25. Mitchell AJ, Benito-León J, González JM, Rivera-Navarro J (2005) Quality of life and its assessment in multiple sclerosis: integrating physical and psychological components of wellbeing. Lancet Neurol 4: 556-566.

26. Montel SR, Bungener C (2007) Coping and quality of life in one hundred and thirty five subjects with multiple sclerosis. Mult Scler 13: 393-401.

27. Pfennings L, Cohen L, Adèr H, Polman C, Lankhorst G, et al. (1999) Exploring differences between subgroups of multiple sclerosis patients in health-related quality of life. J Neurol 246: 587-591.

28. Fjeldstad C, Pardo G, and Bemben M (2008) Quality of life according to duration of disease in women with low disability in multiple sclerosis. International Journal of MS Care 10: 77-80.

29. Mostert S, Kesselring J (2002) Effects of a short-term exercise training program on aerobic fitness, fatigue, health perception and activity level of subjects with multiple sclerosis. Mult Scler 8: 161-168.

30. Oken BS, Kishiyama S, Zajdel D, Bourdette D, Carlsen J, et al. (2004) Randomized controlled trial of yoga and exercise in multiple sclerosis. Neurology 62: 2058-2064.

31. Motl RW, McAuley E, Snook EM, Gliottoni RC (2008) Does the relationship between physical activity and quality of life differ based on generic versus disease-targeted instruments? Ann Behav Med 36: 93-99.

32. Polman $\mathrm{CH}$, Reingold SC, Banwell B, Clanet M, Cohen JA, et al. (2011) Diagnostic criteria for multiple sclerosis: 2010 revisions to the McDonald criteria. Ann Neurol 69: 292-302.

33. Rigby SA, Domenech C, Thornton EW, Tedman S, Young CA (2003) Development and validation of a self-efficacy measure for people with multiple sclerosis: the Multiple Sclerosis Self-efficacy Scale. Mult Scler 9: 73-81.

34. Hobart J, Lamping D, Fitzpatrick R, Riazi A, Thompson A (2001) The Multiple Sclerosis Impact Scale (MSIS-29): a new patient-based outcome measure. Brain 124: 962-973.

35. Riazi A, Hobart JC, Lamping DL, Fitzpatrick R, Thompson AJ (2002) Multiple Sclerosis Impact Scale (MSIS-29): reliability and validity in hospital based samples. J Neurol Neurosurg Psychiatry 73: 701-704.

36. McGuigan C, Hutchinson M (2004) The multiple sclerosis impact scale (MSIS29 ) is a reliable and sensitive measure. J Neurol Neurosurg Psychiatry 75 266-269.

37. Godin G, Shephard RJ (1985) A simple method to assess exercise behavior in the community. Can J Appl Sport Sci 10: 141-146.

38. Gosney JL, Scott JA, Snook EM, Motl RW (2007) Physical activity and multiple sclerosis: validity of self-report and objective measures. Fam Community Health 30: 144-150. 
Citation: Fjeldstad C, Pardo G (2014) Self-efficacy, Physical Activity and QOL in People with MS. J Neurol Neurophysiol 5: 194. doi:10.4172/21559562.1000194

39. Motl RW, McAuley E, Snook EM, Scott JA (2006) Validity of physical activity measures in ambulatory individuals with multiple sclerosis. Disabil Rehabil 28: $1151-1156$.

40. Gutierrez GM, Chow JW, Tillman MD, McCoy SC, Castellano V, et al. (2005) Resistance training improves gait kinematics in persons with multiple sclerosis. Arch Phys Med Rehabil 86: 1824-1829.

41. Motl RW, Snook EM (2008) Physical activity, self-efficacy, and quality of life in multiple sclerosis. Ann Behav Med 35: 111-115.
42. Schmitt MM, Goverover Y, Deluca J, Chiaravalloti N (2013) Self-Efficacy as a Predictor of Self-Reported Physical, Cognitive, and Social Functioning in Multiple Sclerosis. Rehabil Psychol

43. Busse ME, Pearson OR, Van Deursen R, Wiles CM (2004) Quantified measurement of activity provides insight into motor function and recovery in neurological disease. J Neurol Neurosurg Psychiatry 75: 884-888.

44. Ng AV, Kent-Braun JA (1997) Quantitation of lower physical activity in persons with multiple sclerosis. Med Sci Sports Exerc 29: 517-523.

This article was originally published in a special issue, Neurodegenerative Diseases: Symptoms and Therapeutics handled by Editor(s). Dr. Jin J Luo, Temple University School of Medicine, USA 